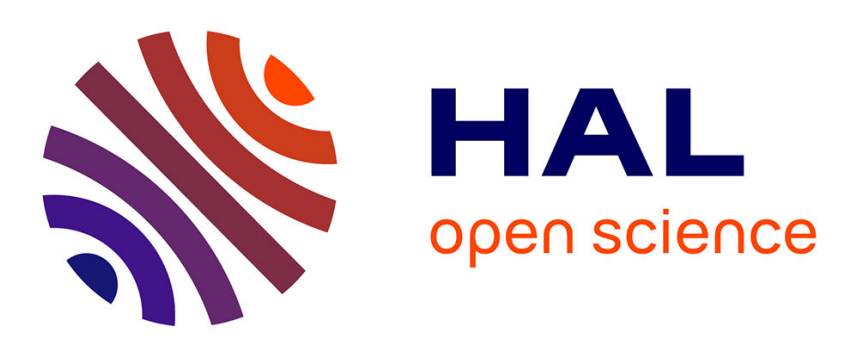

\title{
Recent studies of docking and molecular dynamics simulation for liquid-phase enantioseparations
}

Paola Peluso, Alessandro Dessì, Roberto Dallocchio, Victor Mamane, Sergio

Cossu

\section{- To cite this version:}

Paola Peluso, Alessandro Dessì, Roberto Dallocchio, Victor Mamane, Sergio Cossu. Recent studies of docking and molecular dynamics simulation for liquid-phase enantioseparations. Electrophoresis, In press, 2019, 40, pp.1881-1896. 10.1002/elps.201800493 . hal-02106485

\section{HAL Id: hal-02106485 \\ https://hal.science/hal-02106485}

Submitted on 15 May 2020

HAL is a multi-disciplinary open access archive for the deposit and dissemination of scientific research documents, whether they are published or not. The documents may come from teaching and research institutions in France or abroad, or from public or private research centers.
L'archive ouverte pluridisciplinaire HAL, est destinée au dépôt et à la diffusion de documents scientifiques de niveau recherche, publiés ou non, émanant des établissements d'enseignement et de recherche français ou étrangers, des laboratoires publics ou privés. 
${ }^{1}$ Istituto di Chimica Biomolecolare ICB CNR - Sede Secondaria di Sassari, Traversa La Crucca 3, Regione Baldinca, 07100 Li Punti - Sassari, Italy

${ }^{2}$ Institut de Chimie de Strasbourg, UMR 7177, Equipe LASYROC, 1 rue Blaise Pascal, BP 296 R8, 67008 Strasbourg Cedex, France

*Correspondence: Dr. Paola Peluso, Istituto di Chimica Biomolecolare ICB CNR - Sede Secondaria di Sassari, Traversa La Crucca 3, Regione Baldinca, I-07100 Li Punti - Sassari, Italy

E-mail: paola.peluso@cnr.it

Abbreviations: ADMPC, amylose tris(3,5-dimethylphenylcarbamate); AMBER, assisted model building with energy refinement; CCMPC, cellulose tris(3-chloro-4-methylphenylcarbamate); CCPC, cellulose tris(3-chlorophenylcarbamate); CDCPC, cellulose tris(3,5-dichlorophenylcarbamate); CDMPC, cellulose tris(3,5-dimethylphenylcarbamate); CF, cyclofructan; CHARMM, chemistry at Harvard macromolecular mechanics; CM, carboxymethyl; CMB, cellulose tris(4methylphenylbenzoate); CSP, chiral stationary phase; DC, dielectric constant; EEO, enantiomer elution order; EMO, enantiomer migration order; ESH, explicit $\sigma$-hole; GOLD, genetic optimisation for ligand docking; HB, hydrogen bond; HBA, hydrogen bond acceptor; HBD, hydrogen bond donor; ITC, isothermal titration calorimetry; LGA, Lamarckian genetic algorithm; MD, molecular dynamics; MeOH, methanol; MM, molecular mechanics; MP, mobile phase; NP, normal phase; PABA, $p$ aminobenzoic acid; PEP, positive extra point; PO, polar organic; RMSD, root mean square deviation; SFC, supercritical fluid chromatography.

Keywords: Chiral stationary phase / Docking / Liquid-phase enantioseparation / Molecular dynamics / Molecular recognition 
Liquid-phase enantioseparations have been fruitfully applied in several fields of science. Various

32 applications along with technical and theoretical advancements contributed to increase significantly the

33 knowledge in this area. Nowadays, chromatographic techniques, in particular HPLC on chiral stationary

34 phase, are considered as mature technologies. In the last thirty years, CE has been also recognized as one

35 of the most versatile technique for analytical scale separation of enantiomers. Despite the huge number

36 of papers published in these fields, understanding mechanistic details of the stereoselective interaction

37 between selector and selectand is still an open issue, in particular for high-molecular weight chiral

38 selectors like polysaccharide derivatives. With the ever growing improvement of computer facilities,

39 hardware and software, computational techniques have become a basic tool in enantioseparation science.

40 In this field, molecular docking and dynamics simulations proved to be extremely adaptable to model

41 and visualize at molecular level the spatial proximity of interacting molecules in order to predict

42 retention, selectivity, enantiomer elution order, and profile noncovalent interactions patterns underlying

43 the recognition process. On this basis, topics and trends in using docking and molecular dynamics as

44 theoretical complement of experimental LC and CE chiral separations are described herein. The basic

45 concepts of these computational strategies and seminal studies performed over time are presented, with a

46 specific focus on literature published between 2015 and November 2018. A systematic compilation of

47 all published literature has not been attempted. 


\section{Introduction}

In keeping with chiral recognition mechanisms occurring in biochemical environment, enantioseparation science is based on the concept that a chiral enantiopure molecule (selector) can recognize the enantiomer pair of a chiral analyte (selectand) through noncovalent interactions which underlie the distinction process [1] (Scheme 1).

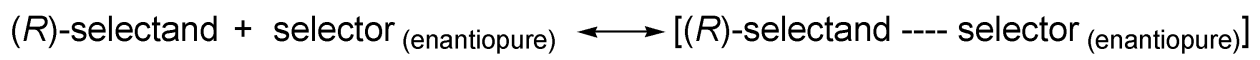

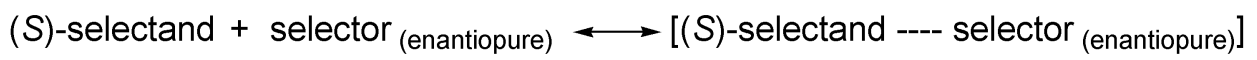

Scheme 1. Equations describing the concept of enantioseparation by means of the reversible and dynamic formation of diastereoisomers between $R / S$ enantiomer pair of selectand and selector.

In LC on chiral stationary phase (CSP), a dynamic process, occurring by means of adsorptiondesorption steps between CSP and mobile phase (MP), enables the transformation of two enantiomers into transient diastereomeric complexes, which are characterized by different chemo-physical properties and different $\Delta \mathrm{G}$ values, $\Delta \Delta \mathrm{G}_{R, S}^{\circ}$ being related to the enantioseparation factor $(\alpha)$ through the equation $\Delta \Delta \mathrm{G}_{R, S}^{\circ}=-\mathrm{RT} \ln \alpha$. Enantiorecognition occurs on the basis of the same principle when chiral selector is introduced in the chromatographic system as an additive to MP, but this technique is rarely used in LC.

Differently, in CE the chiral selector is usually added to the BGE as part of the MP and, therefore, selector-selectand complexes are mobile. As a consequence, in this case two principles govern enantioseparations [2,3]: i) the chromatographic enantioselective recognition, occurring at molecular level, between selector and selectand, and ii) the electrophoretic enantioselective separation which is due to different mobility of the diastereomeric complexes.

In the last decades, both LC and CE techniques have been successfully employed in enantioseparation science [2,4-9]. Despite the huge number of papers published in these fields, the understanding of the stereoselective interaction process is still an open issue. Indeed, multiple noncovalent interactions along with other effects can promote retention and enantioseparation [6]: i) strong long-range interactions involved in the primary non-stereoselective binding, ii) non- 
77 stereoselective adsorption of analytes to the solid support, iii) short-range directional noncovalent 78 interactions [1], underlying the stereoselective binding, which are governed by complementarity of 79 functional groups, like hydrogen bond (HB), $\pi-\pi$ interactions, dipole-dipole stacking and the emergent 80 halogen bond [10,11], iv) steric factors deriving from the spatial arrangement of selector binding site, v) 81 conformational changes of selector induced by selectand binding, vi) hydrophobic effects, and vii) 82 solvation effects. This high level of complexity concerns in particular high-molecular weight selectors 83 such as polysaccharide derivatives.

Chromatographic studies and retention models, spectroscopy methods based on FT-IR and NMR, Xray crystallography analysis, and computational methods, which include both chemoinformatics and molecular modelling, have been developed for several years with the aim of gaining information on

87 binding strength and structure of selector-selectand complexes, and type of involved noncovalent interactions $[1,2,6]$. In particular, computational tools contributed to overcome some disadvantages of using other techniques like low solubility of certain selectors and limited reliability of solid state models to describe complexes in solution, observed for spectroscopy and X-ray crystallography, respectively. systems for which the quantum mechanics (QM) approach is, in general, time-consuming from a computational point of view. MM uses potential-energy functions to model molecules, which consist of spherical atoms connected by springs representing bonds [12,13]. An important aspect of modelling enantioselection concerns the concept of molecular potential energy surface which determines shape and dynamic features of the related molecule. In this regard, two main questions have to be tackled, namely where to locate selectand, in or around the selector [14], and how many selector-selectand complexes must be computed (and sample among all the possible reciprocal orientations) to make the calculation really representative of the experimental system [1]. As response to the questions, docking and molecular dynamics (MD) are exploited to reduce the number of sampling on the potential energy surface and define initial and equilibrium mutual positioning of selector and selectand. Thus, both 
methods are often used as theoretical complement of experimental liquid-phase enantioseparations with the general purpose to visualize the complex associations and provide a molecular-level understanding of structure and dynamics of the CSP, retention mechanisms of analytes, interactions of analytes and CSP, and solvation effects at the CSP interface [15].

On this basis, recent representative applications of both docking and MD simulations in LC and CE enantioseparations are presented herein, covering years 2015-2018 (November). Nevertheless, previous seminal and basic studies in the field are also cited for further information. In this regard, some applications of molecular modelling can be found in some excellent reviews concerning chiral recognition mechanisms $[1,2,6,16,17]$ published in the period 2010-2017. In particular, molecular simulation studies in reversed-phase liquid chromatography [15] and computational studies to rationalize chromatographic EEO [18] have been reviewed in the last years as specific topics in the field. Moreover, it is worth highlighting that seminal papers on atomistic model of enantioselective binding and MD theory in chromatography have been published by Lipkowitz [14,19,20] and Felinger [21], respectively.

Although theoretical details on computational methods are beyond the scope of this review, in the next two paragraphs a brief description of aims and working basis of both docking and MD is provided.

\section{Molecular docking and dynamics in liquid-phase enantioseparations}

Molecular docking is generally used to simulate the interaction between the enantiomer pairs and the active site of the selector in order to predict both energy and geometry of selector-selectand binding. A docking process consists of two general steps, namely conformational search through various algorithms, and scoring or ranking of the docked conformations (selector-selectand mutual orientations) (Fig. 1) [22]. The majority of the studies reported in enantioseparation science have been carried out with AutoDock [23] and AutoDockTools as graphical interface [24]. AutoDock employs Lamarckian Genetic Algorithm (LGA) [23] to identify binding conformations of the selectand, as a flexible ligand, to the selector. Genetic algorithm methods describe the three-dimensional arrangement of the molecules involved in the docking by using geometrical (state) variables which, in this specific case, are selector- 
127 selectand distance, the orientation of selectand with respect to the selector, and the torsional degrees of 128 freedom (number of rotatable bonds) of the selectand enantiomers [25]. The program uses a simplified 129 form of AMBER (Assisted Model Building with Energy Refinement) forcefield (see $\S 3$ ) for the energy 130 calculations, and the free energy of binding is calculated by computing van der Waals and Coulombic 131 energy contributions between all atoms of selector and selectand through an empirical functional form $132[26]$.

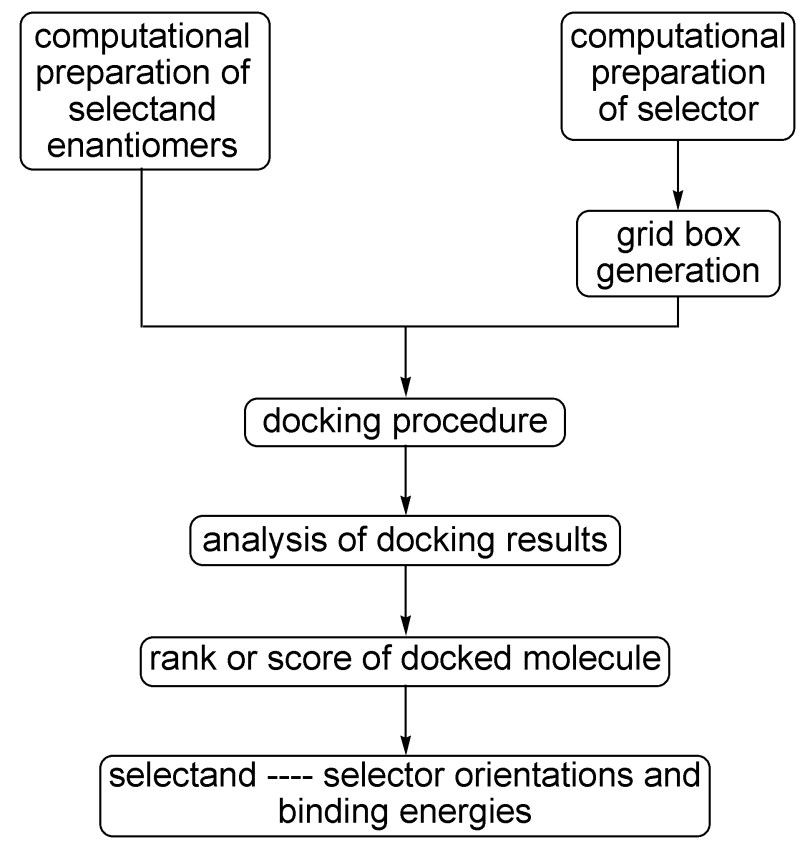

Figure 1. Flow diagram of a general docking protocol.

137 Usually, for application in enantioseparation science, the grid box is set to around $80 \times 80 \times 80 \AA$ with $1380.375 \AA$ spacing. In the computational space profiled by the grid box, each atom type of the chiral 139 analyte is positioned and its interaction energy with each atom of the selector will be computed and 140 assigned to a grid point. All grid points collected for a particular atom-type constitute a map, and during 141 docking the maps are used for extracting interaction energies of the enantiomers with the selector. At the 142 end of docking calculations, several conformers of the enantiomers are obtained and clustered in several 
143 sets. The results are given in terms of the mean binding energy of the clusters or the mean energy of the 144 most populated cluster, and their consistency with the experimental EEO is a basic requirement to 145 develop a reliable predictive model.

146 Introduced in chromatography by Giddings and Eyring in the mid of the last century [27], MD is a 147 simulation that shows how molecules move, vibrate, diffuse, and interact over time [28]. This approach 148 is based on classical mechanical equations of motion related to the enantioseparations system consisting 149 of interacting particles [15]. Several software have been made available, and nowadays commonly used 150 programs for MD simulations include AMBER [29] and CHARMM (Chemistry at HARvard 151 Macromolecular Mechanics) [30], among others. The MD protocol normally consists of six phases: 152 initial assignment, system minimization, heating, cooling, equilibration, and trajectory production (Fig. 153 2) [31].

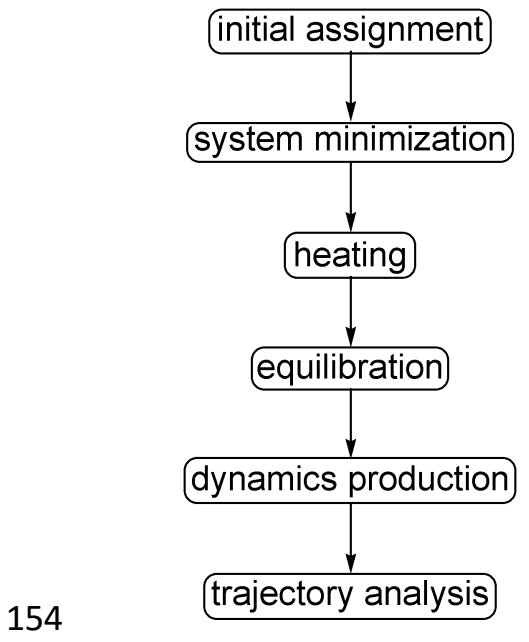

$155 \quad$ Figure 2. Flow diagram of a general MD protocol.

156 On the basis of this sequence, the molecular system is free to run for a period of time and the process 157 is iterated for thousands of steps in order to bring the system to an equilibrium state, saving all the 158 information about the atomic positions, velocities, and other variables as a function of time. The set of 159 data emerging from the MD experiment is called trajectory that profiles positions and velocities of the 160 chiral partners in the system and their variation with time. All the equilibrium and dynamic properties of 161 the system can be calculated from trajectory data set. Interestingly, the root mean square deviation 
(RMSD) of all atoms in a molecule can be plotted against time to summarize the degree of fluctuation

163 for the entire structure.

164 Taking into account that solvent can strongly influence the energy of different complex orientations, in MD simulations solvent can be parametrized by treating it explicitly or implicitly [32]. Explicitsolvent methods introduce solvent molecules by computing interactions between all pairs of solute and 167 solvent atoms, whereas implicit-solvent methods speed up simulations by approximating the discrete solvent as a continuum, thus drastically reducing the number of particles in the system. Moreover, in docking as well as in MDs the proper dielectric constant (DC) value can be used to define the screening effect of solvent on noncovalent interactions, with values ranging from 1 (vacuum) to 80 (water) [26].

Docking calculations and MD simulations can be combined [33,34] to model selectand enantiomers into selectors. First, docking techniques are used to explore a vast conformational space in a short time and scan the possible diastereomeric orientations with the aim of reducing sampling. Then, more accurate MD simulations can be applied when few complexes have been selected. Indeed, a problem of docking concerns the poor flexibility of the selector, which is not permitted to adjust its conformation upon selectand binding, whereas MD treats simulations in a flexible way. However, MD simulations are time-consuming and the length of time that can be saved during a trajectory sampling (usually from ten to hundreds of nanoseconds) is limited by the computer performances and time available. This question can be particularly crucial in modelling large systems. Consequently, in these cases, focused approximations or specific computational techniques are usually applied on a case by case basis. In the next paragraphs, recent applications of docking and MD simulations are discussed on the basis of selector type.

\section{Donor-acceptor chiral selectors}

Donor-acceptor chiral selectors (also called brush-type or Pirkle-type selectors) contain small molecules which are anchored in a silica matrix [35]. These CSPs are able to exert electrostatic interactions based on complementarity like $\mathrm{HB}, \pi-\pi$ interactions and dipole-dipole stacking. In the 
previous decade, some interesting procedures were developed to model enantiomer distinction on Pirkletype CSPs. Cann and co-workers published a series of relevant papers over time concerning the application of MD simulations to explore the solvation at the Whelk-O1 interface [36-38] and the docking modes of different selectands [37]. Interestingly, Gasparrini and co-workers developed a general scheme based on a systematic and automatic "quasi-flexible" docking approach for studying stereoselective recognition mechanisms, validating it on a leucine-containing Pirkle-type selector $[39,40]$. Following previous theoretical studies involving proline-based selector interfaces [41,42], recently Cann and Ashtari employed MD simulations (35-40 ns of simulation time) to model the enantioseparation of six closely related aromatic analytes 1-6 on four polyproline-based CSPs I-IV (Fig.

3) [43].

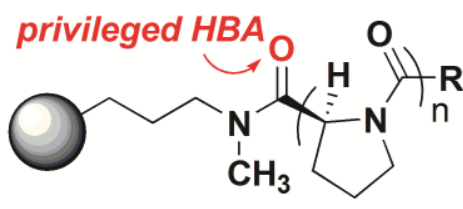
$\begin{array}{lll}\text { CSP I } & \mathrm{n}=2 & \mathrm{R}=\mathrm{C}\left(\mathrm{CH}_{3}\right)_{3} \\ \text { CSP II } & \mathrm{n}=2 & \mathrm{R}=\mathrm{OC}\left(\mathrm{CH}_{3}\right)_{3} \\ \text { CSP III } & \mathrm{n}=3 & \mathrm{R}=\mathrm{C}\left(\mathrm{CH}_{3}\right)_{3} \\ \text { CSP IV } & \mathrm{n}=6 & \mathrm{R}=\mathrm{C}\left(\mathrm{CH}_{3}\right)_{3}\end{array}$<smiles>CC(O)c1ccc2cc3ccccc3cc2c1</smiles>

3<smiles>[R]C(O)c1ccccc1</smiles><smiles>[R]C(O)c1c2ccccc2cc2ccccc12</smiles>

$1 \mathrm{R}=\mathrm{CH}_{3}$

$2 \mathrm{R}=\mathrm{CF}_{3}$

$4 \mathrm{R}=\mathrm{CF}_{3}$

$5 \mathrm{R}=\mathrm{CH}_{3}$

$6 \mathrm{R}=\mathrm{CH}_{2} \mathrm{CH}_{3}$

Figure 3. Structures of Pirkle-type polyproline selectors and aromatic alcohols as selectands in MD simulations [43].

In this study, $48 \mathrm{MD}$ simulations were undertaken, considering each solvent (2) + selector (4) + analyte (6) combination, on a modelled surface consisting of 16 polyprolines, 64 silanol groups, 48 trimethylsilyl end-caps and 128 fixed Si atoms. The theoretical study was performed by considering the effect of two different MPs, namely $n$-hexane/2-propanol, as a nonpolar mixture, and water/methanol, as polar MP, on chiral recognition. In addition, due to focused structure variations in the series of CSPs, the selected chiral selectors allowed an analysis of the impact of oligomer length and terminal group on 
selectivity. On this basis, simulations gave the following information: i) the occurrence of an alternative recognition mechanism in water/methanol compared to the NP elution conditions because of a different

207 HB solvent pattern and a diverse conformational preference of the proline chains; ii) crowding at the interface increases for CSP IV, affecting the arrangement of analyte docking into the surface; iii) the carbonyl oxygens close to the Si layer appeared preferentially involved in chiral recognition as HBA; iv) $\mathrm{HB}$ is the main interaction governing recognition and selectivity coupled with steric hindrance effects at the chiral surface. When possible, the calculated selectivities were compared with the experimental

212 values, finding a good overall agreement (Table 1).

Table 1. Predicted selectivity factors ( $\alpha$ predicted), under NP elution conditions ( $n$-hexane/2-propanol 70:30), derived from MD simulations [43], and available experimental selectivity values ( $\alpha$ experimental) for the enantioseparation of 1-6 on CSPs I and IV

\begin{tabular}{l|l|l}
\hline \multirow{2}{*}{ Analyte } & \multicolumn{2}{|c}{$\alpha$ predicted $(\alpha$ experimental $)$} \\
\cline { 2 - 3 } & CSP I & CSP IV \\
\hline $\mathbf{1}$ & $1.40 \pm 0.13(1.61)$ & $1.92 \pm 0.08(2.60)$ \\
$\mathbf{2}$ & $1.46 \pm 0.10(1.51)$ & $1.77 \pm 0.09(2.08)$ \\
$\mathbf{3}$ & $1.21 \pm 0.11(1.10)$ & $1.09 \pm 0.14(1.00)$ \\
$\mathbf{4}$ & $1.03 \pm 0.16(1.06)$ & $1.00 \pm 0.15(1.10)$ \\
$\mathbf{5}$ & $1.13 \pm 0.15(1.10)$ & $1.08 \pm 0.16(1.00)$ \\
$\mathbf{6}$ & 1.07 & $1.12 \pm 0.16(1.00)$ \\
\hline
\end{tabular}

Topal and co-workers performed both docking and MD simulation (175 ps of simulation time) with AutoDock and AMBER programs, respectively, to investigate chiral recognition mechanism governing the enantioseparation of mandelic acid and 2-phenyl propionic acid on a Pirkle-type CSP synthesized by the authors, finding a good correlation between theoretical and experimental results [44]. ion-exchange CSPs, which have pioneered by Lindner [46], interact with charged analytes via HB or $\pi-\pi$ interactions as other donor-acceptor chiral supports. Nevertheless, long-range ionic interactions between charged selector and selectands also occur. In this field, Natalini, Sardella and co-workers developed a MD simulation protocol (Desmond Molecular Dynamic System 4.0/4.4/5.2 program, $300 \mathrm{~ns}$ of 
224 simulation time) $[47,48]$, which recently has been extensively applied, in collaboration with other 225 groups, to investigate chiral recognition mechanisms and rationalize experimental EEO observed with different Cinchona alkaloids-based CSPs under RP elution [49] and polar organic (PO) [50-52] elution conditions (Table 2).

Table 2. Recent MD simulation studies involving Cinchona alkaloid-based CSPs V-IX [49-52]
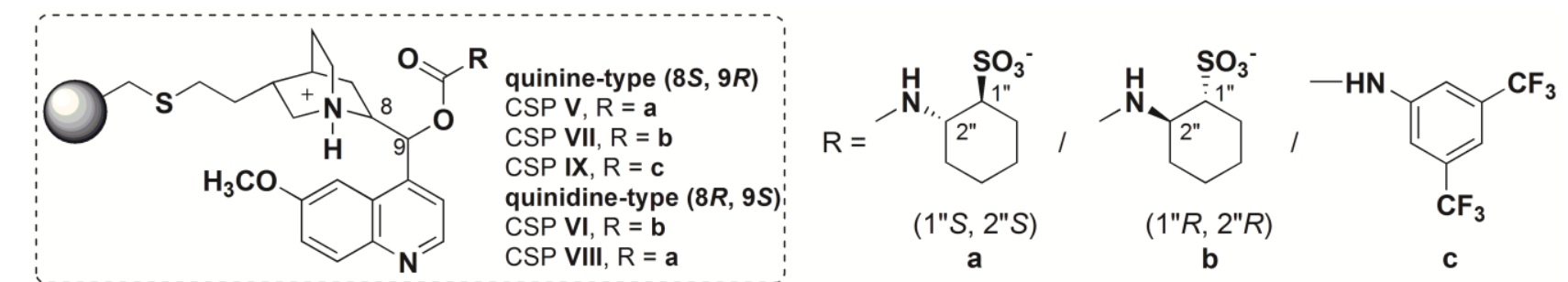

\begin{tabular}{|c|c|c|c|}
\hline CSP & Analyte & MD environment and main results & References \\
\hline V, VI & amminophenylalanine & $\begin{array}{l}\text { custom solvent: } \mathrm{ACN} / \mathrm{MeOH} / \text { water } 49.7: 49.7: 0.6^{\mathrm{a})} \\
\text { main results } \\
\text { - confirmation of } \mathrm{EEO}_{\text {exp }}: \mathrm{D}, \mathrm{D}<\mathrm{D}, \mathrm{L}<\mathrm{L}, \mathrm{D}<\mathrm{L}, \mathrm{L} \\
\text { - active role of achiral element of the CSPs and solvent } \\
\text { - observation of } \mathrm{HBs} \text { between the sulphonic acid group of } \\
\mathrm{CSP} \mathrm{V} \text { and two or three amidic groups of the hydroorotic } \\
\text { fragment }\end{array}$ & $\begin{array}{l}2015 \text { Sardella [49] } \\
\text { (diastereo- and } \\
\text { enantioseparation) }\end{array}$ \\
\hline V-VIII & roxetine enantiomers & $\begin{array}{l}\text { main results } \\
\text { - interaction pattern between paroxetine and CSPS } \\
\text { - confirmation of EEO } \mathrm{O}_{\text {exp }} \text { of paroxetine on CSPS VII and VIII } \\
\text { - limited EEO prediction power of the model for CSPS V and } \\
\text { VI due to its intrinsic inability to consider entropic } \\
\text { contributions }\end{array}$ & $\begin{array}{l}2016 \text { llisz [50] } \\
2018 \text { Carotti [51] }\end{array}$ \\
\hline
\end{tabular}

a) Boc, t-butyloxycarbonyl; $\mathrm{MeOH}$, methanol

The protocol is based on the calculation of three energy descriptors: the interaction energy between the selector unity and the whole discrimination system (named INTER), the interactions energy between selector and selectand (INTER_SA) and the conformational energy of selectand (SELF), relative to its minimum energy derived by the collected MD snapshots. Once energy values are calculated, the matrix is submitted to two k-means clustering runs as a method for cluster analysis to identify families of interactions and the suitable number of clusters which are evaluated and correlated to the experimental 
outcomes [53]. For a realistic reproduction of the CSP, in these studies a surface containing 4 237 mercaptopropyl-functionalized silanols, 8/16 free silanols and $45 \mathrm{Si}$ atom (keeping frozen during MD) 238 was considered for each selector unit. It is worth noting that, in this case, selector being anchored in 239 achiral support, achiral sub-structural elements are also considered on the modelled surface because non240 enantioselective adsorption sites have been found to contribute to retention behaviour.

\section{Polysaccharide derivatives}

Currently, polysaccharide-based CSPs are the most used for LC enantioseparations. These selectors are characterized by a modular polymeric system where molecular, conformational, and supramolecular chirality cooperate to determine the separation outcome [1]. Their structure consists of a glucosyl backbone (cellulose or amylose linkage), derivatized by carbamate or benzoate functionalities with an internal polar layer, and an aromatic layer, functioning as modulator of the electronic properties of the

247 polar layer (Fig. 4). Firstly introduced by Okamoto and co-workers [54], the versatility of 248 polysaccharide derivatives as chiral selectors was improved by Chankvetadze and co-workers by 249 introducing halogen substituents on the phenyl rings [4].

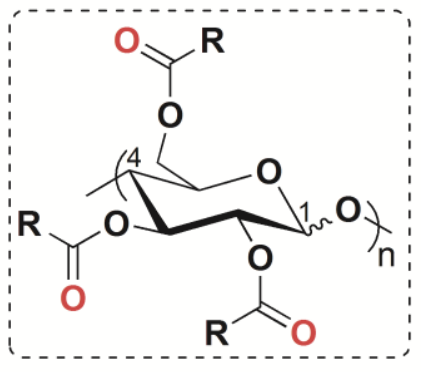

cellulose-based $(\beta-1,4$ linkage) amylose-based ( $\alpha-1,4$ linkage) polar layer, aromatic layer

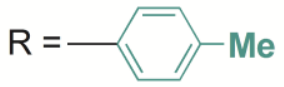

CMB (cellulose)

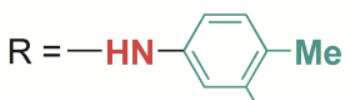

CCMPC (cellulose) $\mathrm{Cl} \quad$ CCPC (cellulose)

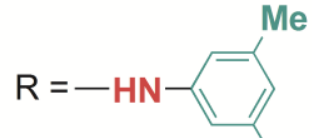

CDMPC (cellulose) Me

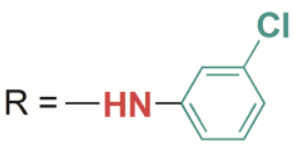<smiles>[R]CNc1cccc(C)c1</smiles>

ADMPC (amylose) Me

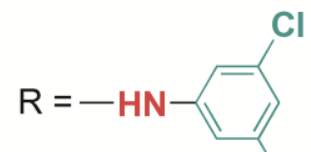

CDCPC (cellulose) $\mathrm{Cl}$

Figure 4. Structures of some polysaccharide-based selectors modelled by docking and MD simulations. related enantioseparations have been published by the groups of Okamoto [55-57], Franses [58-60], and

254 Grinberg $[61,62]$. In addition, further interesting modelling studies were performed on polysaccharidederivatives and published in the last years [63-67]. In Figure 4, the structures of some polysaccharide 
selectors modelled over time are reported. All these studies contributed to understand some aspects of

257 the chiral recognition: i) the chromatographic behaviour can depend on the polymer backbone as well as 258 on the type of side chain; ii) attractive interactions play an important role in the recognition as well as 259 the steric fit of the analyte inside the chiral cavity, where polar carbamate groups are considered as 260 important chiral adsorbing sites. Nevertheless, understanding the recognition mechanism at molecular 261 level is still demanding due to the intrinsic complexity of these selectors.

262 In Table 3, a summary of some representative docking and MD studies published in the period 20152632018 is reported [68-78]. Firstly, in modelling polysaccharides, an important issue concerns the 264 preparation of a built polymer which is representative of the 'real' polysaccharide derivative. On the 265 basis of the studies of Okamoto's group, cellulose tris(3,5-dimethylphenylcarbamate) (CDMPC) and 266 amylose tris(3,5-dimethylphenylcarbamate) (ADMPC) have been characterized by a left-handed (3/2) 267 and 4/3 helix, respectively. Usually, oligomeric fragments composed of 12 monomers are used, despite 268 sometimes studies involving shorter oligomers have been reported probably to reduce computational 269 time. In this regard, recently Liu and co-workers reported a molecular docking studies by using trimer 270 fragments to model CDMPC, ADMPC and cellulose tris(3-chloro-4-methylphenylcarbamate) 271 (CCMPC) and the enantiomers of napropamide 7 as analytes [68]. On this basis, the study partly 272 explained the variations of experimental EEOs observed with the three CSPs under supercritical fluid 273 chromatography $(\mathrm{SFC})$ conditions $\left(\mathrm{CO}_{2} /\right.$ modifier $=$ alcohol or $\left.\mathrm{ACN}\right)$. Indeed, variations of the $\mathrm{HB}$ 274 features between enantiomers of 7 and each CSP were considered to justify the EEO of $S-R$ and $R-S$ 275 observed on amylose and cellulose CSPs, respectively. In other recent studies, fragment of 5-6 276 monomers have been considered. Ali and co-workers studied both diastereo- and enantioseparation of a 277 large series of dipeptides 8 on ADMPC by using molecular docking $[69,70]$. Later, the same authors also 278 modelled by docking the recognition of the four stereoisomer of 5-bromo-3-ethyl-3-(4279 nitrophenyl)piperidine-2,6-dione 9 on ADMPC [71]. 
Table 3. Recent docking and MD simulation studies involving polysaccharide-based selectors [68-78]

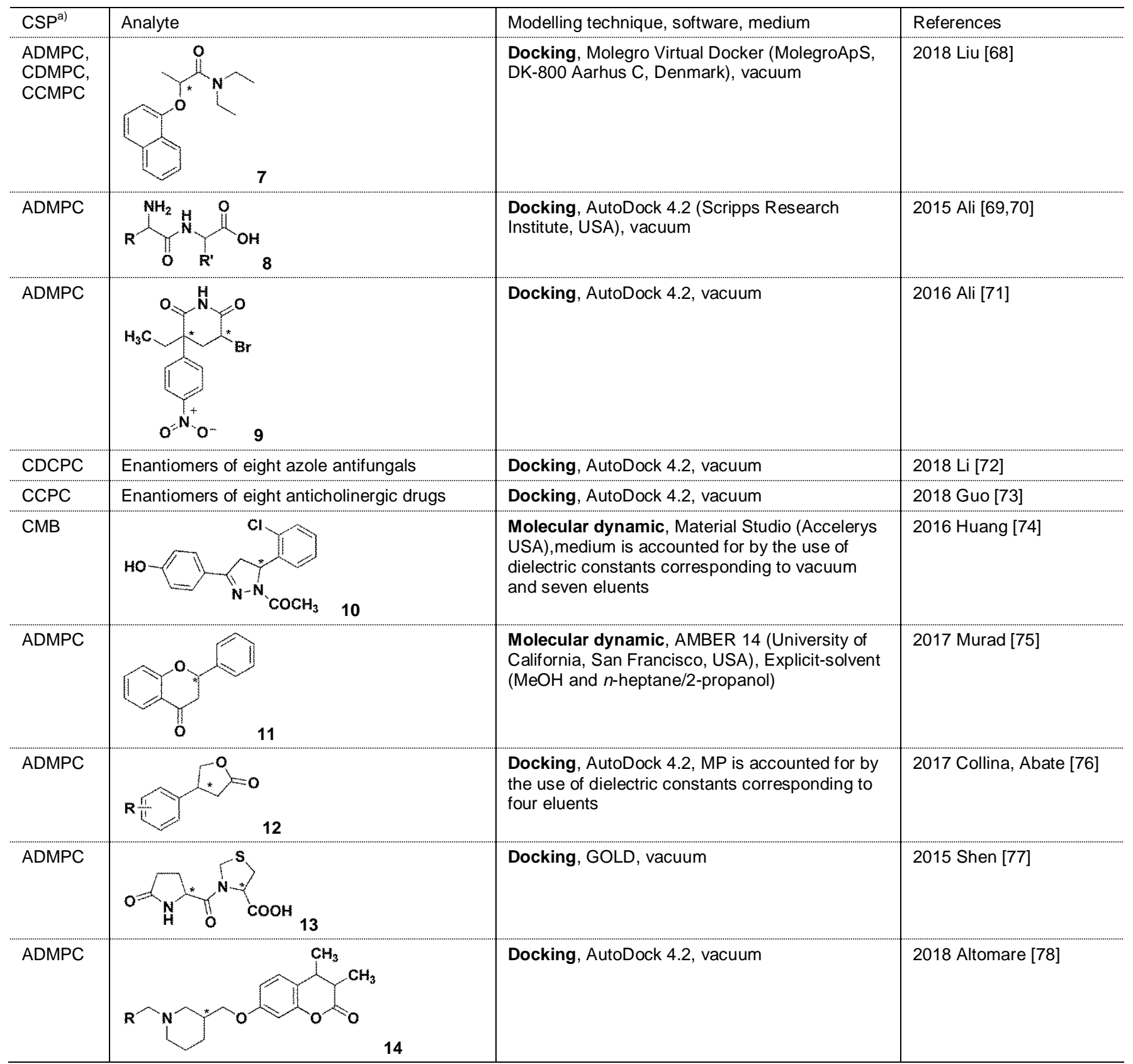

a) ADMPC, amylose tris(3,5-dimethylphenylcarbamate); CCMPC cellulose tris(3-chloro-4-methylphenylcarbamate); CCPC cellulose tris(3chlorophenylcarbamate); CDCPC cellulose tris(3,5-dichlorophenylcarbamate); CDMPC cellulose tris(3,5-dimethylphenylcarbamate); CMB cellulose tris(4-methylphenylbenzoate). molecular docking [72]. The variation of the binding energies (average energy of the best cluster with the lowest docking energy) of the complexes formed by $R$ - and $S$-enantiomers was in agreement with the 
observed enantioselectivity under NP elution conditions (Table 4). Guo and co-workers reported a 291 docking study, modelling cellulose tris(3-chlorophenylcarbamate) (CCPC) as selector and the 292 enantiomers of eight anticholinergic drugs (atropine sulfate, phenylcyonate, dipivefrine hydrochloride, 293 tropicamide, homatropine methylbromide, oxybutynin, scopolamine hydrobromide, benzhexol 294 hydrochloride) [73].

Table 4. Correlation between the variation of the binding energies (average energy of the best cluster with the lowest docking energy) of the complexes formed by $R$ - and $S$-enantiomers and selectivity factors on CDCPC $^{\text {a) }}$ under NP elution

\begin{tabular}{l|l|l}
\hline Analyte & $|\Delta \Delta \mathrm{E}[\mathrm{kcal} / \mathrm{mol}]|^{\mathrm{b})}$ & $\alpha$ \\
\hline Butoconazole & 0.71 & 1.95 \\
Ornidazole & 0.55 & 1.46 \\
Sulconazole & 0.47 & 1.29 \\
Enilconazole & 0.38 & 1.27 \\
Isoconazole & 0.35 & 1.25 \\
Econazole & 0.33 & 1.24 \\
Ketoconazole & 0.26 & 1.21 \\
Futrimazole & 0.11 & 1.06 \\
\hline
\end{tabular}

a) CDCPC, cellulose tris(3,5-dichlorophenylcarbamate)

b) $|\Delta \Delta \mathrm{E}[\mathrm{kcal} / \mathrm{mol}]|=\left|\Delta \mathrm{E}_{R}-\Delta \mathrm{E}_{S}\right|$

297 The composition of the MP can have a very important effect on chiral recognition, therefore the effect of solvent should be considered in theoretical computational studies. Huang and co-workers modelled the enantioselection of a chiral pyrazole derivative $\mathbf{1 0}$ on cellulose tris(4-methylbenzoate)

(CMB) by means of MDs (100ps of simulation time) [74]. In the study, a 12-monomer fragment was 301 built to model CMB, with the terminal monomers replaced by methyl groups, and seven mixtures were 302 used as custom solvents. DC values were set by the authors to represent the experimental conditions as 303 follows: $n$-hexane/ethanol $(70 / 30)(\mathrm{DC}=9.06), n$-hexane/2-propanol $(60 / 40)(\mathrm{DC}=8.58)$, pure ethanol $304(\mathrm{DC}=25.80)$ and pure 2-propanol $(\mathrm{DC}=18.62)$. In addition, three reference solvent conditions, vacuum 305 (DC = 1), pure $n$-hexane (1.89) and water (81.00) were also considered in order to explore the solvent 306 effect systematically. The computational experiments showed that the solvent effect has an important 307 influence on selector-selectand binding energies. Consequently, in polar solvents (DC $\geq 8.58)$ the $S$ 308 enantiomer $\cdots$ CSP complex appeared more stable that the $R$-enantiomer $\cdots$ CSP complex, according to 
the experimental EEO of $R-S$ reported for $\mathbf{1 0}$ by using PO solvent or NP with an alcohol content $\geq 30 \%$. Murad and coworkers used quantum mechanics (QM)/MM and MD simulations to model the enantiomers of flavanone 11 on a 12-mer ADMPC (100 ns of simulation time) [75]. It is worth noting that the hybrid QM/MM approach is not uncommon in this field because it combines the accuracy of QM and speed of MM, allowing for the study of large molecules in solution. In this study, custom solvents were introduced explicitly corresponding to $\mathrm{MeOH} 100 \%$ and heptane/2-propanol as experimental eluents. Significantly, the simulations showed that the lifetime of HBs formed between ADMPC and flavanone enantiomers are able to reproduce the EEO observed in the experiments performed under PO and NP conditions. Abbate, Collina and co-workers described a series of molecular docking experiments which were performed to justify the constant $S$ - $R$ EEO observed for all enantiomeric pairs of four 3-aryl-substituted- $\gamma$-butyrolactones $\mathbf{1 2}$ on the ADMPC under NP and PO elution conditions [76]. In this study, the MP composition was simulated by using DC values corresponding to the experimental MPs. The mean docking energy proved to be consistent with the chromatographic results and, for each enantiomeric pair, the higher calculated binding energy corresponded to the first eluted $(S)$-enantiomer.

In principle, molecular docking can be performed in vacuum without modelling MP effect. In this case, despite the fact that the variations of energy predicted by docking in vacuum could be different compared to experimental results in solution, a good agreement can be also found. In this regard, Shen and co-workers performed a comparative docking by using ADMPC and cyclodextrins (CDs) as selectors and the enantiomers of pidotimod $\mathbf{1 3}$ as analytes. The difference of HBs, van der Waals, and internal torsional tension energy between the enantiomers and CSPs were found to be the leading causes of chiral recognition [77]. Altomare and coworkers modelled by docking the enantioseparation of three coumarin derivatives 14 on a 12-mer ADMPC [78]. Interestingly, with the aim of achieving a plausible low energy conformation, the authors subjected the ADMPC fragment to a short MD, assembling the 
solvated model in an orthorhombic box filled with methanol molecules to mimic the MP (experimental $\mathrm{MP}=\mathrm{MeOH} / \mathrm{ACN})$

\section{Cyclodextrins}

CDs are cyclic oligosaccharides formed by D-glucose units with $\alpha-1,4$ linkages. These molecules are

337 characterized by a hollow toroid-shape, lipophilic inside and hydrophilic outside, where secondary 2338 and 3-hydroxyl groups are located at the wider rim, while primary 6-hydroxyl groups at the narrower 339 rim. Due to the possibility to modify chemically the hydroxyl groups, a large number of CD derivatives 340 are commercially available and immobilized to solid supports.

341

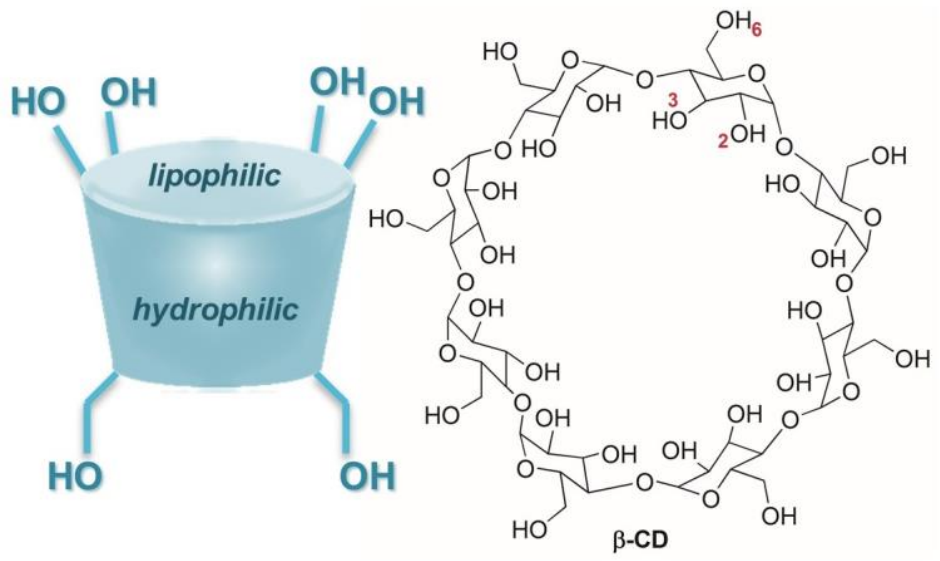

Figure 5. General scheme of CD hollow toroid-shape and structure of $\beta-C D$.

Despite the fact that 'external' complexes between CD derivatives and guest molecules have been observed [79], the main complexation mode occurs via inclusion between an apolar part of the guest molecule by hydrophobic interactions in the cavity, and polar interactions at the polar rim of the CD [80]. Several noncovalent interactions underlie recognition processes like HBs, $\pi-\pi$ and hydrophobic interactions, dipole-dipole stacking, van der Waals and dispersion forces. On one hand, the fact that CDs can be studied in solution allowed NMR to give a great contribution to understand their recognition mechanism [81]. On the other hand the complexity of the possible recognition pattern which govern inclusion, or external contacts, make molecular modelling a versatile tool also in this case. 
Current trends in molecular modelling applied to the study of CDs have been recently reviewed [82]

352 with a specific focus on drug delivery matrixes and intelligent nanodevices such as CD-based molecular 353 motors. Moreover, in the last years Alvira performed a deep investigation on MD simulation approaches 354 to model amino acid enantiodiscrimination by using $\alpha-, \beta-, \gamma$-CDs and a series of CD derivatives [83355 85]. Recently, Wang and co-workers modelled the enantioseparation of flavonone with $\beta$-CDs by MDs, 356 introducing the mixture $\mathrm{MeOH} /$ water 1:1 (RPLC conditions) as custom solvent [86]. Starting from a 357 different approach, López-Nicolás and co-workers modelled the contacts between methyl jasmonate 358 stereoisomers and methyl- $\beta-\mathrm{CD}$ by molecular docking, as theoretical complement of the experimental 359 enantioseparation performed by adding the selector to the MP, under RPLC conditions [87].

360 Due to some features like availability, low toxicity ad UV absorbance along with good solubility, 361 CDs have been largely applied as chiral selector in CE. Several studies concerning docking of CE 362 enantiorecognition have been published in the last years [88-92]. In this regard, it is worth mentioning 363 that despite the fact that the prerequisite for separation of enantiomers is their enantioselective 364 interaction with a chiral selector, the EEO in CE does not necessarily correlate with the enantioselective 365 recognition, as it occurs in LC on CSP, because of the additional contribution of the electrophoretic 366 enantioseparation mechanism [2]. Moreover, as argued by Chankvetadze, CE is one of the most 367 sensitive tools for detecting very weak enantioselective noncovalent interactions because of the very 368 high separation efficiency, and energy difference between the diastereomeric complexes at the level of 369 few $\mathrm{kJ} / \mathrm{mol}$ is sufficient for observing baseline separation of enantiomers. Nevertheless, this advantage 370 implicates that a reliable evaluation of such small energy differences by means of molecular modelling 371 requires the selection of the proper force field, charge state of selector and selectand, and proper 372 parametrization to account for solvent effect [2].

373 Using CDs as chiral selector in CE allows chiral recognition mechanisms to be studied in solution by 374 spectroscopic methods and separation techniques under similar conditions [2,81]. Recently, interesting 
investigations of the complexation between CDs and enantiomers have been published, which are performed by means of a multidisciplinary approach based on the use of CE, NMR and molecular simulations (Table 5). You and co-workers performed the enantioseparation of four chiral drugs (2amino-1-phenylethanol, 1-(4-methoxyphenyl)-2-(methylamine)ethanol, salbutamol sulfate, sotalol hydrochloride) by $\mathrm{CE}$ using both $\beta-\mathrm{CD}$ and carboxymethyl- $\beta$-cyclodextrin $(\mathrm{CM}-\beta-\mathrm{CD})$ that exhibited the best separation efficiency [93]. In this study, isothermal titration calorimetry (ITC), NMR and molecular docking were used to gain information about recognition mechanism. On this basis, it was found that hydrophobic interactions, electrostatic interactions and HBs underlie the enantioselection induced by the CM- $\beta-C D$. Orlandini, Furlanetto and co-workers developed a method for the enantioseparation of sulpiride (15) enantiomers by CE with the addition of two types of CDs to the BGE, namely the negatively charged sulfate- $\beta-C D$ sodium salt and a neutral CD [80]. A multidisciplinary approach based on both NMR and MD was used to investigate recognition mechanism. MDs was performed with $3 \mathrm{~ns}$ of production time, in implicit solvent. On one hand, MD simulation suggested, in agreement with CE experiments, a relationship between the gain in potential energy and migration time. On the other hand, NMR showed the inclusion of the benzene sulphonamide moiety of the analyte inside the hydrophobic cavity of the CDs. Very recently, the same author studied the separation mechanism involved in CD-MEKC analysis of ambrisentan (16) enantiomers by means of the combined CE/NMR/MD approach. The study provided information on the aggregates, inclusion complexes and noncovalent interactions underlying the separation system [94]. Salgado and co-workers used again NMR spectroscopy and MD (100 ns of production time) to investigate structure and energy of the binding complexes between the enantiomers of clenpenterol 17 and two CDs, namely $\beta$-CDs and heptakis(2,3-di- $O$-acetyl)- $\beta$-cyclodextrin (HAD- $\beta$-CD) [95]. The study showed that the inclusion mode of $\mathbf{1 7}$ is dependent on CD structure and that intermolecular HBs are mediated by bridging water 
molecules. Moreover, computed interaction energies proved to account for both enantioseparation and enantiomer migration order (EMO) reversal observed by changing $\beta-\mathrm{CD}$ to HAD- $\beta-\mathrm{CD}$.

Table 5. Recent combined molecular modelling - NMR - CE investigations by using CDs as selectors [3,80,93-95]

\begin{tabular}{|c|c|c|c|}
\hline$C D^{a)}$ & Analyte & Modelling technique, software, medium & References \\
\hline $\begin{array}{l}\beta-C D \\
C M-\beta-C D\end{array}$ & $\begin{array}{l}\text { 2-amino-1-phenylethanol, } \\
\text { 1-(4-methoxyphenyl)-2-(methylamine)ethanol, } \\
\text { salbutamol sulfate, } \\
\text { sotalol hydrochloride }\end{array}$ & $\begin{array}{l}\text { Docking, AutoDock } 4.2 \text { (Scripps } \\
\text { Research Institute, USA), vacuum }\end{array}$ & 2015 You [93] \\
\hline $\begin{array}{l}\text { sulfate- } \beta-C D \\
\text { sodium salt }+ \\
\text { neutral CD }\end{array}$ & $\mathrm{H}_{3} \mathrm{C}$ & $\begin{array}{l}\text { Molecular dynamics, AMBER (University } \\
\text { of California, San Francisco, USA), implicit } \\
\text { solvent }\end{array}$ & $\begin{array}{l}2015 \text { Orlandini, } \\
\text { Furlanetto [80] }\end{array}$ \\
\hline$\gamma-C D$ & & $\begin{array}{l}\text { Molecular dynamics, AMBER, implicit } \\
\text { solvent }\end{array}$ & $\begin{array}{l}2017 \text { Orlandini, } \\
\text { Furlanetto [94] }\end{array}$ \\
\hline & 17 & & \\
\hline$\beta-C D$ & & Molecular dynamics, AMBER, water box & 2018 Scriba [3] \\
\hline$\gamma-C D$ & & & \\
\hline$H S-\beta-C D$ & & & \\
\hline & 18 & & \\
\hline
\end{tabular}

a) CM- $\beta$-CD, carboxymethyl- $\beta$-cyclodextrin; HAD- $\beta$-CD, heptakis(2,3-di-O-acetyl)- $\beta$-cyclodextrin; $H S-\beta-C D$, heptakis(6-O-sulfo)- $\beta$-CD.

Later, Scriba and coworkers investigated the influence on EMO of medetomidine (18) of both cavity size and substitution pattern of CDs used as selectors in CE environment [3]. Also, in this case, both NMR and MD simulations (100 ns simulation time) contribute to rationalize the binding mechanism, 404 showing that for $\beta$-CD and $\gamma-\mathrm{CD}$ the phenyl moiety of medetomidine enter the cavity from the wider 405 secondary rim of the CDs, while the protonated imidazole ring points toward the bulk solvent. In the complex with heptakis(6- $O$-sulfo)- $\beta-\mathrm{CD}(\mathrm{HS}-\beta-\mathrm{CD})$, the protonated imidazolium moiety appears to be positioned inside the CD cavity interacting with the sulfate groups in position 6 of the glucose monomer. 
Cyclofructans (CFs), which have been introduced in separation sciences by Armstrong and coworkers [96], are cyclic oligosaccharides composed of $\beta-2,1$ linked D-fructofuranose units. Showing an opposite pattern compared to $\mathrm{CDs}$, CFs have internal HB interactions and do not present hydrophobic 414 cavities. By using the simple p-aminobenzoic acid (PABA), Armstrong, Sun and co-workers [97] 415 demonstrated a $\mathrm{pH}$ driven complexation between CF6 (containing 6 fructose units) and PABA by using 416 a combined MD-NMR approach.

417 Among macrocyclic glycopeptides, the most important from an analytical perspective are 418 vancomycin, ristocetin A, teicoplanin and teicoplanin aglycone. The selector structure consists of 419 interconnected amino acid-based macrocycles, each macrocycle containing two aromatic rings. These 420 glycopeptides form a C-shaped basket, several interactions underlying recognition mechanisms like 421 HBs, $\pi-\pi$, dipole-dipole, ion-dipole, ionic and hydrophobic interactions [6]. Pinto, Fernandez and co422 workers modelled the contacts between thirty-one chiral xanthonic analytes and four macrocyclic 423 glycopeptides by docking [98]. The theoretical study showed that each glycopeptide featured different 424 patterns. Ali and co-workers modelled the enantioseparation of four quinolones on teicoplanin by 425 molecular docking, and HBs and $\pi-\pi$ interactions were found to be the major forces for chiral 426 recognition [99].

427 Recently MD simulations were also exploited to study contact between enantiomers and molecularly 428 imprinted polymers [100], single wall carbon nanotubes [101], and chiral molecular micelles [102,103]. 429 Moreover, docking was also used to model enantiodiscrimination events involving chiral ionic liquids, 430 as MP additives [104].

\section{Molecular dynamics simulations of $\sigma$-hole-driven enantioseparations}

In the last years, our groups investigated the factors governing HPLC enantioseparation of 
434 studies, we have demonstrated the contribution of halogen $(\mathrm{XB})$ and chalcogen $(\mathrm{ChB})$ bonds for the 435 enantiorecognition of analytes on CDMPC [11,12,108-111].

$436 \mathrm{XBs}$ and $\mathrm{ChBs}$ are noncovalent interactions belonging to the family of $\sigma$-hole bonds which occur 437 between the electrophilic region ( $\sigma$-hole) of the halogen or chalcogen atom ( $\sigma$-hole donor) attached to 438 one molecule and the nucleophilic region of the interacting partner ( $\sigma$-hole acceptor) [112]. Applications 439 involving both $\mathrm{XB}$ and $\mathrm{ChB}$ have rapidly grown in the last years and important advancements appeared 440 in supramolecular chemistry, biology and catalysis $[113,114]$.

441 The electrophilic nature of halogens and chalcogens is due to the anisotropic distribution of the 442 electron density around these atoms [115]. Computational techniques have an essential role in 443 investigating this family of interactions, but conventional molecular mechanics (MM) force fields fail to 444 describe the XB because they did not account for the anisotropic distribution of the electron density. 445 Therefore, several MM approaches describing $\sigma$-hole in halogens were proposed in the last years [116]. 446 Early 2010's, three groups have almost simultaneously shown that the $\sigma$-hole can be represented as a 447 positively charged dummy-atom. Ibrahim modelled the $\sigma$-hole as a massless point charge, called 448 positive extra point (PEP), placed on top of the halogen atoms and the optimal position of the PEP was 449 determined to be equal to the atomic radius of the halogen atom [117,118]. Sironi and co-workers 450 proposed almost exactly the same model where the pseudo-atom has a nonzero mass [119,120]. 451 Introduced by Hobza and co-workers, the molecular mechanical explicit $\sigma$-hole (ESH) was constructed 452 as a massless point charge and the ESH parameters were fixed in terms of ESH-halogen distance and 453 units of the positive charge [121,122]. This approach of adding a partial positive charge in the region of 454 the $\sigma$-hole along the $\mathrm{C}-\mathrm{X}$ axis was successfully implemented in the AMBER force fields package, 455 allowing significant improvement of the geometries and interaction energies for halogen-bonded 456 complexes. It has been later applied by Jorgensen and co-workers to enhance the OPLS-AA force field 457 for the description of halogens [123] and sulfur charge anisotropy [124]. The improved AMBER 
program was successfully employed by different groups for MD studies in biological [125] and supramolecular systems [126,127]. Recently, XBs were also parametrized in CHARMM [128] and GROMOS [129] force fields.

On this basis, recently we used the ESH concept to model XB in CDMPC- and ADMPChalobipyridine complexes by MD simulations (10 ns of simulation time) [109,110] (Fig. 6).
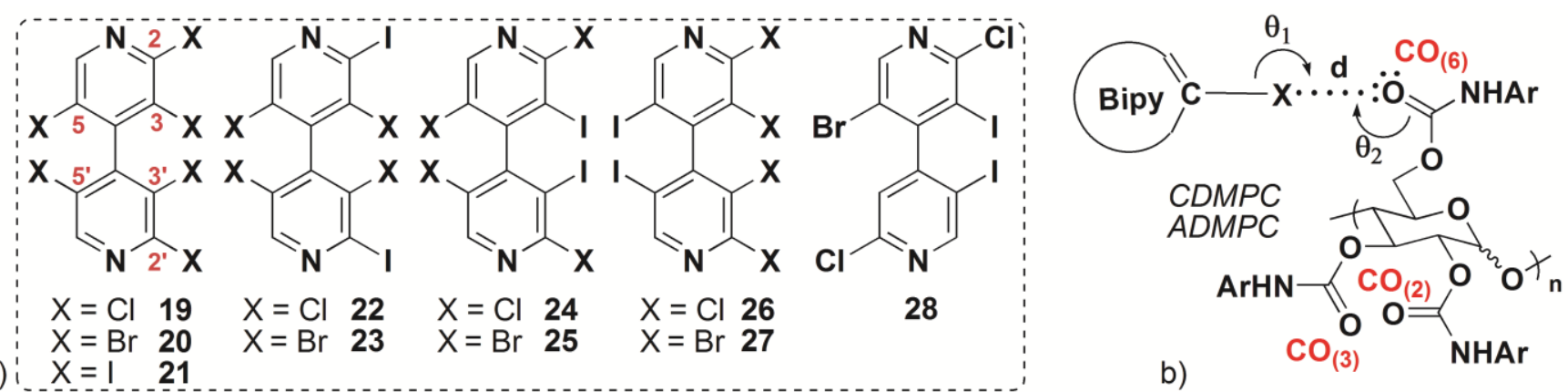

463

464

465

466

467

468

469

470

471

472

473

474

475

476

477

Figure 6. Structures of polyhalo-4,4'-bipyridines 19-28 used in MD studies and geometrical parameters $\left(\mathrm{d}, \theta_{1}, \theta_{2}\right)$ of the XB complex between halogenated 4,4'-bipyridines and polysaccharide-based CSPs.

In all these studies, a massless dummy atom connected to $\mathrm{I}, \mathrm{Cl}$ and $\mathrm{Br}$ was introduced manually, by using distance and charge values as described by Hobza and co-workers [122]. The parameters used for $\mathrm{Cl}, \mathrm{Br}$, I were $1.0,1.3,1.6 \AA$, and $0.1,0.2,0.3$ units of positive charge for the extra point (EP), respectively. In order to keep the total charge of the molecule unchanged, an equivalent negative charge was manually added to each halogen atom. On the other hand, molecular models of 9-mer CDMPC and ADMPC were constructed in order to investigate on the binding sites functioning as $\sigma$-hole acceptors. The geometrical parameters analysed were i) the distance (d) between halogens and XBA centres, ii) the angle $\left(\theta_{1}\right)$ formed by aromatic carbon, halogen, and oxygen atom $\left(\mathrm{C}-\mathrm{X} \cdots \mathrm{O}\right.$, reference value $\left.180^{\circ}\right)$, and iii) the angle $\left(\theta_{2}\right)$ formed by halogens, carbonyl oxygen and carbonyl carbon $(X \cdots O=C$, reference value $120^{\circ}$ ) (Fig. 6b). In particular, any distance shorter than the sum of the van der Waals radii of oxygen and halogen may be considered as an implication of $\mathrm{XB}$. It is worth noting that, in general, $\theta_{1}$ angles ranging from $160^{\circ}$ to $180^{\circ}$ are considered acceptable to decide if the interaction corresponds to a XB. 
The potential contacts occurring in the course of the MDs on 4,4'-bipyridine 19-28 were examined.

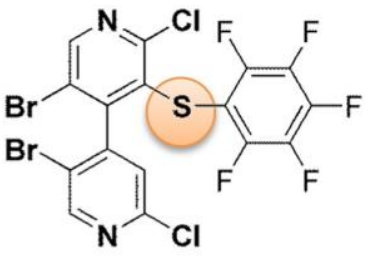

a) and the CSP carbonyls as acceptors (Fig. 7) [111]. 29

b)

Indeed, taking into account the dynamic feature of the enantioseparation event, the distances between each of the six halogens as donor recognition sites and 14 points $(\mathrm{N}, \mathrm{O}, \mathrm{H})$ located on each monomers of the 9-mers CDMPC and ADMPC were statistically analyzed. The carbonyls $\mathrm{CO}_{6}$ and $\mathrm{CO}_{3}$ (Fig. 6b) were found to be the most frequent recognition sites in the simulations of halobipyridines exhibiting high experimental selectivity. In several cases, the results of the statistical evaluation of the observed contacts in the course of the four MDs were consistent with the experimental EEOs. The EEOs assigned on a model based exclusively on XB interactions were in agreement with the experimental EEO in 18 simulations out of 32 , with an overall success rate of $56.2 \%$. It is worth noting that the rate increases to 75\% considering the CDMPC exclusively, whereas it decreases to $37.5 \%$ for ADMPC. This evidence could be related to the fact that on ADMPC other entropy-driven forces had been found to control enantiorecognition along XB [109]. Consequently, it was likely that an exclusive XB model does not adequately describe XB-driven enantioseparations on the amylose-based CSP.

MD simulations were also performed to model the interaction modes of compound 29 (Fig. 7a) with the CDMPC, hypothesizing the occurrence of a chalcogen bond between the electrophilic sulfur on 29
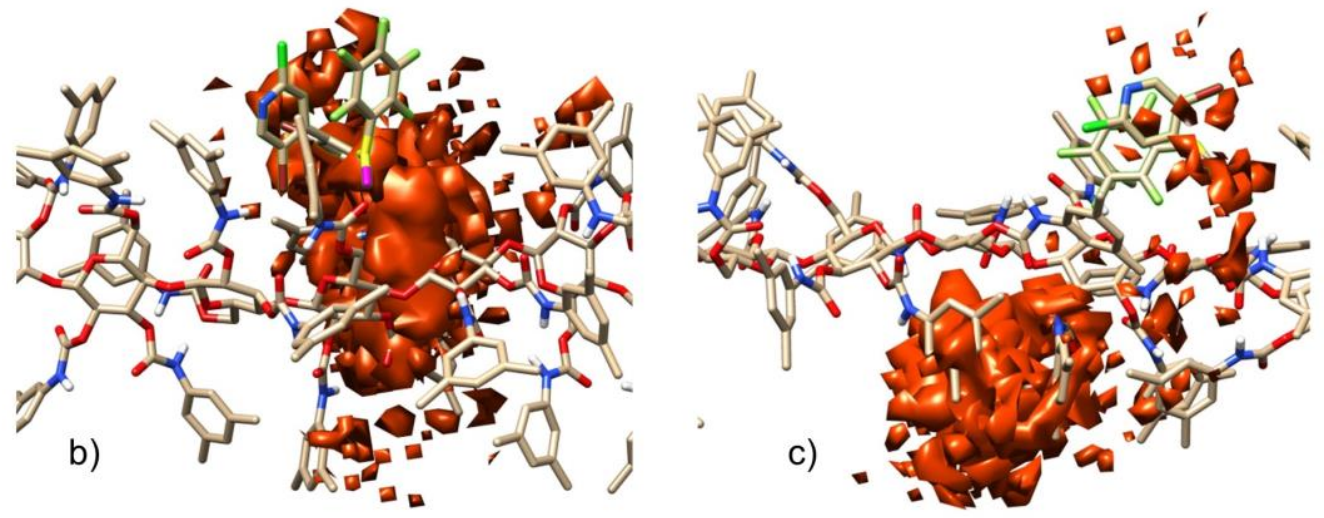

Figure 7. Structure of compound 29 and comparison of the occupancy graphs of the MD simulations of CDMPC-29 complexes over $10 \mathrm{~ns}$ : a) $\mathrm{CDMPC} /(P)-29(\mathrm{MAD})$ vs b) $\mathrm{CDMPC} /(P)-29$ (without MAD). 
A massless dummy atom (MAD) connected to sulfur was introduced manually, by using distance (1.6

498

$\AA$ ) and charge ( 0.2 units of positive charge) fixed arbitrarily. In all simulations performed by using the MAD correction, contacts between sulfur and the carbonyl groups of the carbamate moieties of the CSP were observed. The occupancy analysis was also performed in order to evaluate which regions of space were highly populated by the analyte over $10 \mathrm{~ns}$ of simulation time. Interestingly, by using the MAD, the first eluted enantiomer $(M)$-29 showed occupancy volumes in the outer region of the CSP, whereas for the second eluted enantiomer $(P)-\mathbf{2 9}$ occupancy volumes were also generated in the inner regions of the polymer (Fig. 7b). On the contrary, the occupancy volumes for the enantiomer $(P)$-29 were shown to move toward the outer regions of the polymer when the MAD correction was not applied (Fig. 7c).

\section{Concluding remarks}

Nowadays, the use of MM methods suitable for studying large molecular systems and the ongoing improvement in both software and hardware tools are making molecular modelling more and more faithful to simulate enantiomer distinction. A multidisciplinary approach based on the use of orthogonal techniques, involving also molecular modelling, usually enables researchers to obtain reliable mechanistic information. In addition, there is a tendency to develop computational software and platform increasingly friendly. On the one hand, some key steps appear to be crucial in modelling the spatial proximity of selector and selectand in solvated environment: i) choice of force fields suitable for both selectors and selectands, in particular when high-molecular weight selectors have to be treated; ii) the theoretical environment needs to be consistent with the experimental conditions, for example in terms of solvent composition; iii) the design of both selector and selectand involved in the simulation should be made taking into account the responses expected by the simulations. Indeed, the comparison of the computational responses for structurally related series of analytes and selectors can provide useful information about the impact on recognition of focused frameworks and structural variations; iv) all choices should always emerge from a balanced compromise between the need to obtain theoretical 
521 results as reliable as possible and approximations, which are dependent on computational time and 522 performances, and complexity of the modelled chromatographic system.

523 In this context, docking and MD strategies provide several types of information on chiral recognition:

524 i) molecular level justifications of observed chromatographic behaviours, in particular the experimental 525 EEO; ii) visualization and binding energies of selector-selectand associations; iii) definition of type, 526 topology, and geometrical parameters (distance, angle) of noncovalent interactions underlying the 527 complexes. Importantly, in our recent studies, MD simulations contributed to develop a recognition 528 model for the emergent $\sigma$-hole bond-driven enantioseparations.

529 Finally, it is worth noting that knowledge of chiral recognition mechanisms allows researchers to 530 improve selector-selectand system performance with the aim of optimizing selectivity [130], all the 531 while paving the way to emerging fields of supramolecular separation science like chiral sensing [131] 532 and other chiral surface related recognition phenomena [132].

$533 \quad$ The authors declared no conflict of interest.

$534 \quad 9$ References

535 [1] Lämmerhofer, M., J. Chromatogr. A 2010, 1217, 814-856.

536 [2] Chankvetadze, B., J. Chromatogr. A 2018, 1567, 2-25.

537 [3] Krait, S., Salgado, A., Chankvetadze, B., Gago, F., Scriba, G. K. E., J. Chromatogr. A 2018, $538 \quad 1567,198-210$.

539 [4] Chankvetadze, B., J. Chromatogr. A 2012, 1269, 26-51.

540 [5] Li, X., Chang, C., Wang, X., Bai, Y., Liu, H., Electrophoresis 2014, 35, 2733-2743.

541 [6] Scriba, G. K. E., J. Chromatogr. A 2016, 1467, 56-78.

542 [7] Speybrouck, D., Lipka, E., J. Chromatogr. A 2016, 1467, 33-55.

543 [8] Peluso, P., Mamane, V., Aubert, E., Cossu, S., Electrophoresis 2017, 38, 1830-1850.

544 [9] Fanali, S., Electrophoresis 2017, 38, 1822-1829.

545 [10] Peluso, P., Mamane, V., Aubert, Cossu, S., J. Chromatogr. A 2014, 1345, 182-192. 
[11] Peluso, P., Mamane, V., Aubert, E., Dessì, A., Dallocchio, R., Dore, A., Pale, P., Cossu, S., J. Chromatogr. A 2016, 1467, 228-238.

[12] Vanommeslaeghe, K., Guvench, O., MacKerell Jr., A. D., Curr. Pharm. Des. 2014, 20, 32813292.

[13] Adcock, S. A., McCammon, J. A., Chem. Rev. 2006, 106, 1589-1615.

[14] Lipkowitz, K. B., J. Chromatogr. A 2001, 906, 417-442.

[15] Lindsey, R. K., Rafferty, J. L., Eggimann, B. L., Siepmann, J. I., Schure, M. R., J. Chromatogr. A 2013, 1287, 60-82.

[16] Scriba, G. K. E., Chromatographia 2012, 75, 815-838.

[17] Stavrou, I. J., Agathokleous, E. A., Kapnissi-Christodoulo, C. P., Electrophoresis 2017, 38, 786-819.

[18] Sardella, R., Ianni, F., Macchiarulo, A., Pucciarini, L., Carotti, A., Natalini, B., Mini-Rev. Med. Chem. 2018, 18, 88-97.

[19] Lipkowitz, K. B., J. Chromatogr. A 1995, 694, 15-37.

[20] Lipkowitz, K. B., Acc. Chem. Res. 2000, 33, 555-562.

[21] Felinger, A., J. Chromatogr. A 2008, 1184, 20-41.

[22] Karthikeyan, M., Vyas, R., Practical Chemoinformatics, Springer India 2014, pp. 195-197.

[23] Morris, G. M., Goodsell, D. S., Halliday, R. S., Huey, R., Hart, W. E., Belew, R. K., Olson, A. J., J. Comput. Chem. 1998, 19, 1639-1662.

[24] Morris, G. M., Huey, R., Lindstrom, W., Sanner, M. F., Belew, R. K., Goodsell, D. S., Olson, A. J., J. Comput. Chem. 2009, 30, 2785-2791.

[25] Ravichandran, S., Collins, J. R., Singh, N., Wainer, I. W., J. Chromatogr. A 2012, 1269, $218-$ 225.

[26] Tsai, C. S., An Introduction to Computational Biochemistry, Wiley-Liss, Inc., New York 2002, pp. 292-295 (MDs) and pp. 320-322 (docking). 
[27] Giddings, J. C., Eyring, H., J. Phys. Chem. 1955, 59, 416-421.

[28] Young, D. C., Computational Drug Design, John Wiley \& Sons, Inc. Hoboken, New Jersey 2009, p. 294.

[29] Case, J. T. B. D. A., Betz, R. M., Cerutti, D. S., Cheatham III, T. E., Darden, T. A., Duke, R. E., Giese, T. J., Gohlke, H., Goetz, A.W., Homeyer, N., Izadi, S., Janowski, P., Kaus, J., Kovalenko, A., Lee, T. S., LeGrand, S., Li, P., Luchko, T., Luo, R., Madej, B., Merz, K. M., Monard, G., Needham, P., Nguyen, H., Nguyen, H. T., Omelyan, I., Onufriev, A., Roe, D. R., Roitberg, A., Salomon-Ferrer, R., Simmerling, C. L., Smith, W., Swails, J., Walker, R. C., Wang, J., Wolf, R. M., Wu, X., York, D. M., Kollman, P. A., AMBER 2015, University of California, San Francisco, 2015.

[30] Brooks, B. R., Bruccoleri, R. E., Olafson, B. D., States, D. J., Swaminathan, S., Karplus, M., J. Comput. Chem. 1983, 4, 187-217.

[31] Patodia, S., Bagaria, A., Chopra, D., J. Phys. Chem. Biophys. 2014, 4, 1-4.

[32] Anandakrishnan, R., Drozdetski, A., Walker, R. C., Onufriev, A. V., Biophys. J. 2015, 108, $1153-1164$.

[33] Alonso, H., Bliznyuk, A. A., Gready, J. E., Med. Res. Rev. 2006, 26, 531-568.

[34] Hospital, A., Goñi, J. R., Orozco, M., Gelpí, J. L., Adv. App. Bioinf. Chem. 2015, 8, 37-47.

[35] Fernandes, C., Phyo, Y. Z., Silva, A. S., Tiritan, M. E., Kijjoa, A., Pinto, M. M. M., Sep. Purif. Rev. 2018, 47, 89-123.

[36] Zhao, C. F., Cann, N. M., J. Chromatogr. A 2006, 1131, 110-129.

[37] Zhao, C. F., Cann, N. M., Anal. Chem. 2008, 80, 2426-2438.

[38] Hall, K., Ashtari, M., Cann, N. M., J. Chem. Phys. 2012, 136, 114705, doi: 10.1063/1.3693516.

[39] Alcaro, S., Gasparrini, F., Incani, O., Mecucci, S., Misiti, D., Pierini, M., Villani, C., J. Comput. Chem. 2000, 21, 515-530. 
[40] Alcaro, S., Gasparrini, F., Incani, O., Caglioti, L., Pierini, M., Villani, C., J. Comput. Chem. 2007, 28, 1119-1128.

[41] Ashtari, M., Cann, N. M., J. Chromatogr. A 2011, 1218, 6331-6347.

[42] Ashtari, M., Cann, N. M., J. Chromatogr. A 2012, 1265, 70-87.

[43] Ashtari, M., Cann, N. M., J. Chromatogr. A 2015, 1409, 89-107.

[44] Çakmak, R., Ercan, S., Sünkür, M., Yilmaz, H., Topal, G., Org. Commun. 2017, 10, $216-227$.

[45] Lämmerhofer, M., Anal. Bioanal. Chem. 2014, 406, 6095-6103.

[46] Lämmerhofer, M., Lindner, W., J. Chromatogr. A 1996, 741, 33-48.

[47] Sardella, R., Carotti, A., Gioiello, A., Lisanti, A., Ianni, F., Lindner, W., Natalini, B., J. Chromatogr. A 2014, 1339, 96-102.

[48] Sardella, R., Lisanti, A., Carotti, A., Blasi, P., Lindner, W., Natalini, B., J. Sep. Sci. 2014, 37, 2696-2703.

[49] Ianni, F., Carotti, A., Marinozzi, M., Marcelli, G., Di Michele, A., Sardella, R., Lindner, W., Natalini, B., Anal. Chim. Acta 2015, 885, 174-182.

[50] Grecsó, N., Kohout, M., Carotti, A., Sardella, R., Natalini, B., Fülöp, F., Lindner, W., Péter, A., Ilisz, I., J. Pharm. Biomed. Anal. 2016, 124, 164-173.

[51] Sardella, R., Macchiarulo, A., Urbinati, F., Ianni, F., Carotti, A., Kohout, M., Lindner, W., Péter, A., Ilisz, I., J. Sep. Sci. 2018, 41, 1199-1207.

[52] Ianni, F., Pucciarini, L., Carotti, A., Gioiello, A., Galarini, R., Natalini, S., Sardella, R., Lindner, W., Natalini, B., J. Chromatogr. A 2018, 1557, 20-27.

[53] Guo, Z., Mohanty, U., Noehre, J., Sawyer, T.K., Sherman, W., Krilov, G., Chem. Biol. Drug Des. 2010, 75, 348-359.

[54] Okamoto, Y., Yashima, E., Angew. Chem. Int. Ed. 1998, 37, 1020-1043.

[55] Yashima, E., Yamada, M., Kaida, Y., Okamoto, Y., J. Chromatogr. A 1995, 694, 347-354.

[56] Yamamoto, C.; Yashima, E.; Okamoto, Y. J. Am. Chem. Soc. 2002, 124, 12583-12589. 
[57] Yamamoto, C., Yashima, E., Okamoto, Y., Bull. Chem. Soc. Jpn. 1999, 72, 1815-1825.

[58] Kasat, R. B., Wang, N. H. L., Franses, E. I., Biomacromolecules 2007, 8, 1676-1685.

[59] Kasat, R. B., Wang, N. H. L., Franses, E. I., J. Chromatogr. A 2008, 1190, 110-119.

[60] Kasat, R. B., Franses, E. I., Wang, N. H. L., Chirality 2010, 22, 565-579.

[61] O’Brien, T., Crocker, L., Thompson, R., Thompson, K., Toma, P. H., Conlon, D. A., Feibush, B., Moeder, C., Bicker, G., Grinberg, N., Anal. Chem. 1997, 69, 1999-2007.

[62] Ma, S., Tsui, H. -W., Spinelli, E., Busacca, C. A., Franses, E. I., Wang, N. H. L., Wu, L., Lee, H., Senanayake, C., Yee, N., Gonella, N., Fandrick, K., Grinberg, N., J. Chromatogr. A 2014, $1362,119-128$.

[63] Booth, T. D., Wainer, I. W., J. Chromatogr. A 1996, 737, 157-169.

[64] Ye, Y. K., Bai, S., Vyas, S., Wirth, M. J., J. Phys. Chem. B 2007, 111, 1189-1198.

[65] Li, Y., Liu, D., Wang, P., Zhou, Z., J. Sep. Sci. 2010, 33, 3245-3255.

[66] Kim, B. -H., Lee, S. U., Moon, D. C., Chirality 2012, 24, 1037-1046.

[67] Ortuso, F., Alcaro, S., Menta, S., Fioravanti, R., Cirilli, R., J. Chromatogr. A 2014, 1324, 7177.

[68] Zhao, L., Xie, J., Guo, F., Liu, K., Chirality 2018, 30, 661-669.

[69] Ali, I., Haque, A., Al-Othman, Z. A., Al-Warthan, A., Asnin, L., Sci. China Chem. 2015, 58, 519-525.

[70] Ali, I., Sahoo, D. R., Al-Othman, Z. A., Al-Warthan, A., Asnin, L., Larsson, B., J. Chromatogr. A 2015, 1406, 201-209.

[71] Ali, I., Lone, M. N., Suhail, M., Al-Othman, Z. A., Al-Warthan, A., RSC Adv. 2016, 6, 1437214380.

[72] Zhu, B., Zhao, F., Yu, J., Wang, Z., Song, Y., Li, Q., New J. Chem. 2018, 42, 13421-13429.

[73] Li, M., Zhang, B., Yu, J., Wang, J., Guo, X., New J. Chem. 2018, 42, 11724-11731.

[74] Hu, G., Huang, M., Luo, C., Wang, Q., Zou, J. -W., J. Mol. Graph. Model. 2016, 66, 123-132. 
[75] Zhao, B., Oroskar, P. A., Wang, X., House, D., Oroskar, A., Oroskar, A., Jameson, C., Murad, S., Langmuir 2017, 33, 11246-11256.

[76] Rossi, D., Nasti, R., Collina, S., Mazzeo, G., Ghidinelli, S., Longhi, G., Memo, M., Abbate, S., J. Pharm. Biomed. Anal. 2017, 144, 41-51.

[77] Dou, X., Su, X., Wang, Y., Chen, Y., Shen, W., Chirality 2015, 27, 802-808.

[78] Pisani, L., Rullo, M., Catto, M., de Candia, M., Carrieri, A., Cellamare, S., Altomare, C. D., J. Sep. Sci. 2018, 41, 1376-1384.

[79] Gogolashvili, A., Tatunashvili, E., Chankvetadze, L., Sohajda, T., Szemann, J., Salgado, A., Chankvetadze, B., Electrophoresis 2017, 38, 1851-1859.

[80] Melani, F., Pasquini, B., Caprini, C., Gotti, R., Orlandini, S., Furlanetto, S., J. Pharm. Biomed. Anal. 2015, 114, 265-271.

[81] Salgado, A., Chankvetadze, B., J. Chromatogr. A, 2016, 1467, 95-144.

[82] Quevedo, M. A., Zoppi, A., J. Incl. Phenom. Macrocycl. Chem. 2018, 90, 1-14.

[83] Alvira, E., Tetrahedron: Asymmetry 2013, 24, 1198-1206.

[84] Alvira, E., Tetrahedron: Asymmetry 2015, 26, 853-860.

[85] Alvira, E., Chemical Physics Letters 2017, 679, 31-37.

[86] Li, X., Yao, X., Xiao, Y., Wang, Y., Anal. Chim. Acta 2017, 990, 174-184.

[87] Matencio, A., Bermejo-Gimeno, M. J., García-Carmona, F., López-Nicolás, J. M., Phytochem. Anal. 2017, 28, 151-158.

[88] Suliman, F. O., Elbashir, A. A., Schmitz, O. J., J. Incl. Phenom. Macrocycl. Chem. 2015, 83, 119-129.

[89] Arsad, S. R., Maarof, H., Ibrahim, W. A. W., Aboul-Enein, H. Y., Chirality 2016, 28, 209-214.

[90] Yang, X., Du, Y., Feng, Z., Liu, Z., Li, J., J. Chromatogr. A 2018, 1559, 170-177.

[91] Li, J., Yu, T., Xu, G., Du, Y., Liu, Z., Feng, Z., Yang, X., Xi, Y., Liu, J., J. Chromatogr. A 2018, 1559, 178-185. 
[92] Yang, X., Yan, Z., Yu, T., Du, Y., Chen, J., Liu, Z., Xi, Y., Anal. Bioanal. Chem. 2018, 410, 5889-5898.

[93] Li, L., Li, X., Luo, Q., You, T., Talanta 2015, 142, 28-34.

[94] Pasquini, B., Melani, F., Caprini, C., Del Bubba, M., Pinzauti, S., Orlandini, S., Furlanetto, S., J. Pharm. Biomed. Anal. 2017, 144, 220-229.

[95] Salgado, A., Tatunashvili, E., Gogolashvili, A., Chankvetadze, B., Gago, F., Phys. Chem. Chem. Phys. 2017, 19, 27935-27939.

[96] Sun, P., Wang, C., Breitbach, Z. S., Zhang, Y., Armstrong, D. W., Anal. Chem. 2009, 81, 10215-10226.

[97] Wang, L., Li, C., Yin, Q., Zeng, S., Sun, C., Pan, Y., Armstrong, D. W., Tetrahedron 2015, 71, $3447-3452$.

[98] Phyo, Y. Z., Cravo, S., Palmeira, A., Tiritan, M. E., Kijjoa, A., Pinto, M. M. M., Fernandez, C., Molecules 2018, 23, 142, doi: 10.3390/molecules23010142.

[99] Ali, I., Suhail, M., Asnin, L., Chirality 2018, doi: 10.1002/chir.23024.

[100] Sobiech, M., Żołek, T., Luliński, P., Maciejewska, D., Talanta 2016, 146, 556-567.

[101] Lee, O. -S., J. Comput. Sci. 2016, 15, 60-64.

[102] Morris, K. F., Billiot, E. J., Billiot, F. H., Hoffman, C. B., Gladis, A. A., Lipkowitz, K. B., Southerland, W. M., Fang, Y., Chem. Physics 2015, 457, 133-146.

[103] Morris, K. F., Billiot, E. J., Billiot, F. H., Ingle, J. A., Zack, S. R., Krause, K. B., Lipkowitz, K. B., Southerland, W. M., Fang, Y., J. Dispersion Sci. Tech. 2018, 39, 45-54.

[104] Feder-Kubis, J., Flieger, J., Tatarczak-Michalewska, M., Płazińska, A., Madejska, A., SwatkoOssord, M., RSC Adv. 2017, 7, 32344-32356.

[105] Peluso, P., Mamane, V., Aubert, E., Cossu, S., J. Chromatogr. A 2012, 1251, 91-100.

[106] Peluso, P., Mamane, V., Aubert, E., Cossu, S., J. Sep. Sci. 2013, 36, 2993-3003.

[107] Peluso, P., Mamane, V., Aubert, E., Cossu, S., J. Sep. Sci. 2014, 37, 2481-2489. 
[108] Peluso, P., Mamane, V., Cossu, S., Chirality 2015, 27, 667-684.

[109] Peluso, P., Mamane, V., Dallocchio, R., Dessì, A., Villano, R., Sanna, D., Aubert, E., Pale, P., Cossu, S., J. Sep. Sci. 2018, 41, 1247-1256.

[110] Dallocchio, R., Dessì, A., Solinas, M., Arras, A., Cossu, S., Aubert, E., Mamane, V., Peluso, P., J. Chromatogr. A 2018, 1563, 71-81.

[111] Peluso, P., Gatti, C., Dessì, A., Dallocchio, R., Weiss, R., Aubert, E., Pale, P., Cossu, S., Mamane, V., J. Chromatogr. A 2018, 1567, 119-129.

[112] Desiraju, G. R., Ho, P. S., Kloo, L., Legon, A. C., Marquardt, R., Metrangolo, P., Politzer, P., Resnati, G., Rissanen, K., Pure Appl. Chem. 2013, 85, 1711-1713.

[113] Cavallo, G., Metrangolo, P., Milani, R., Pilati, T., Priimagi, A., Resnati, G., Terraneo, G., Chem. Rev. 2016, 116, 2478-2601.

[114] Mahmudov, K. T., Kamran, T., Kopylovich, M. N., Guedes da Silva, M. F. C., Pombeiro, A. J. L., Dalton Trans. 2017, 46, 10121-10138.

[115] Politzer, P., Murray, J. S., Clark T., Phys. Chem. Chem. Phys. 2013, 15, 11178-11588.

[116] Koláŕ, M. H., Hobza, P., Chem. Rev. 2016, 116, 5155-5187.

[117] Ibrahim, M. A. A., J. Comput. Chem. 2011, 32, 2564-2574.

[118] Ibrahim, M. A. A., J. Mol. Model. 2012, 18, 4625-4638.

[119] Rendine, S., Pieraccini, S., Forni, A., Sironi, M., Phys. Chem. Chem. Phys. 2011, 13, 1950819516.

[120] Franchini, D., Dapiaggi, F., Pieraccini, S., Forni, A., Sironi, M., Chem. Phys. Lett. 2018, 712, 89-94.

[121] Kolář, M., Pavel Hobza, P., J. Chem. Theory Comput. 2012, 8, 1325-1333.

[122] Kolár̆, M., Hobza, P., Bronowska, K., Chem. Commun. 2013, 49, 981-983.

[123] Jorgensen, W. L., Schyman, P., J. Chem. Theory Comput. 2012, 8, 3895-3901. 
719 [124] Yan, X. C., Robertson, M. J., Tirado-Rives, J., Jorgensen, W. L., J. Phys. Chem. B 2017, 121, $720 \quad 6626-6636$.

721 [125] Zhou, Y., Wang, Y., Li, P., Huang, X.-P., Qi, X., Du, Y., Huang, N., ACS Chem. Med. Lett. $722 \quad 2018,9,1019-1024$.

723 [126] Lim, J. Y. C., Marques, I., Thompson, A. L., Christensen, K. E., Félix, V., Beer, P. D., J. Am. $724 \quad$ Chem. Soc. 2017, 139, 3122-3133.

725 [127] Lim, J. Y. C., Marques, I., Félix, V., Beer, P. D., Angew. Chem. Int. Ed. 2018, 57, 584-588.

726 [128] Gutiérrez, I. S., Lin, F.-Y., Vanommeslaeghe, K., Lemkul, J. A., Armacost, K. A., Brooks III, 727 C. L., MacKerell, A. D., Biorg. Med. Chem. 2016, 24, 4812-4825.

728 [129] Nunes, R., Vila-Viçosa, D., Machuqueiro, M., Costa, P. J., J. Chem. Theory Comput. 2018, 14, 729 $5393-5392$

730 [130] Schurig, V., Molecules 2016, 21, 1535; doi:10.3390/molecules21111535.

731 [131] Manoli, K., Magliulo, M., Torsi, L., in: Schurig, V. (Ed.), Differentiation of Enantiomers II, 732 Springer International Publishing, Switzerland 2013, pp. 133-176.

733 [132] Lang, J. C., Armstrong, D. W., Curr. Opin. Colloid Interface Sci. 2017, 32, 94-107. 